\title{
Análise da Temperatura Superficial da Lagoa Mangueira na Passagem de Frentes Frias a partir de Dados de Radiância do Sensor MODIS
}

\author{
Marilei Bender Xavier ${ }^{1}$, Sílvia Beatriz Alves Rolim ${ }^{2}$, Gervásio Annes Degrazia ${ }^{3}$ e Waterloo Pereira Filho ${ }^{4}$ \\ ${ }^{1}$ Departamento de Matemática, Universidade de Santa Cruz do Sul (UNISC), RS. \\ ${ }^{2}$ Centro de Pesquisas Espaciais em Sensoriamento Remoto e Meteorologia, Universidade Federal do Rio Grande do Sul \\ (UFRGS). \\ ${ }^{3}$ Programa de Pós-Graduação em Física e Meteorologia, Universidade Federal de Santa Maria, Santa Maria, RS. \\ (UFSM). \\ ${ }^{4}$ Departamento de Geociências, Universidade Federal de Santa Maria(UFSM),- Santa Maria, RS.
}

Copyright 2014, SBGf - Sociedade Brasileira de Geofísica

Este texto foi preparado para a apresentação no VI Simpósio Brasileiro de Geofísica, Porto Alegre, 14 a 16 de outubro de 2014. Seu conteúdo foi revisado pelo Comitê Técnico do VI SimBGf, mas não necessariamente representa a opinião da SBGf ou de seus associados. É proibida a reprodução total ou parcial deste material para propósitos comerciais sem prévia autorização da SBGf.

\section{Resumo}

Dados de sensoriamento remoto constituem uma importante fonte de obtenção da temperatura de superfície em sistemas aquáticos, uma vez que são capazes de cobrir áreas nem sempre abrangidas por estações convencionais de coleta de dados meteorológicos. Este trabalho aborda a análise comparativa da temperatura superficial de uma lagoa costeira, na passagem de sistemas frontais em dois períodos distintos. A resposta da temperatura de superfície foi medida a partir do produto MOD11Temperatura de Superfície e Emissividade, do sensor Moderate Resolution Imaging Spectroradiometer (MODIS), a bordo da plataforma orbital EOS-TERRA. A temperatura de superfície apresentou maior variação nas presenças de ventos fortes e temperaturas ambientes altas para os períodos estudados. A presente análise mostra que a temperatura de superfície da lagoa diminui após a passagem de frentes fria e está relacionada com o campo de vento originado neste processo.

\section{Introdução}

As medidas de grandezas geofísicas em ambientes naturais podem revelar e prever o efeito de impactos ambientais em particulares ecossistemas caracterizados por uma escala temporal geológica de curta duração. Os ecossistemas aquáticos que apresentam pequenas extensões, como lagoas que ocorrem na interface entre os oceanos e regiões continentais de terra firme (região costeira), possuem uma dinâmica própria e são sensíveis às mudanças meteorológicas provocadas por sistemas frontais (SF) (Tundisi et al., 2010; Mohseni; Mohseni et al., 2003). A temperatura de superfície nestes corpos d'água sofre alterações com a passagem de um SF em diferentes épocas do ano. Alguns parâmetros são alterados com a passagem destes sistemas, como por exemplo, a temperatura ambiente, a velocidade e a direção do vento. Atualmente, não se sabe a extensão da mudança ou influência destes parâmetros meteorológicos com a temperatura superficial em período quente do ano ou um período frio e o quanto vai influenciar o ecossistema aquático com a passagem de SF. Estes fenômenos transitórios desestabilizam a coluna d'água e como conseqüência podem desestabilizar os fatores bióticos do ecossistema (Tundisi et al., 2010). Neste sentido, as observações das temperaturas superficiais, mediadas em uma escala de tempo, podem revelar padrões importantes de mudanças em lagos em diferentes épocas do ano. O presente estudo emprega imagens orbitais para se medir de forma remota a temperatura superficial da lagoa Mangueira. Estas observações são realizadas durante a passagem de sistemas frontais no sul do Brasil. Estudos mostram a mudança em lagos, mas não a análise comparativa de interação da temperatura superficial com os parâmetros meteorológicos na passagem dos SF. Para a realização deste trabalho foi empregada a estimativa da temperatura de superfície do produto MOD11(Temperatura de Superfície e Emissividade) do sensor MODIS e dados meteorológicos medidos na estação de Santa Vitória do Palmar, Estado do Rio Grande do Sul RS. O Produto MOD11 calcula a temperatura de superfície terrestre em função da radiância espectral e das medidas de emissividades nas bandas 31(10,78 a $11,28 \mu \mathrm{m})$ e $32(11,77$ a $12,27 \mu \mathrm{m})$ do infravermelho termal.

\section{Área de Estudo}

A lagoa Mangueira (Figura 1) é um ecossistema de água doce, constituído principalmente por um corpo de água raso, apresentando profundidades que variam entre 1,5 e $6 \mathrm{~m}$ (Delaney,1965). Ela possui uma forma alongada com $92 \mathrm{~km}$ de comprimento e com uma largura oscilando entre 2 e $10 \mathrm{~km}$. A sua área aproximada é de $820 \mathrm{~km}^{2}$ e se estende na zona costeira do sul do Brasil, sendo localizada entre os municípios de Santa Vitória do Palmar e Rio Grande. Esta lagoa constitui uma parte do Sistema Hidrológico da Reserva do Taim, abrangendo uma área total de $2254 \mathrm{Km}^{2}$. 


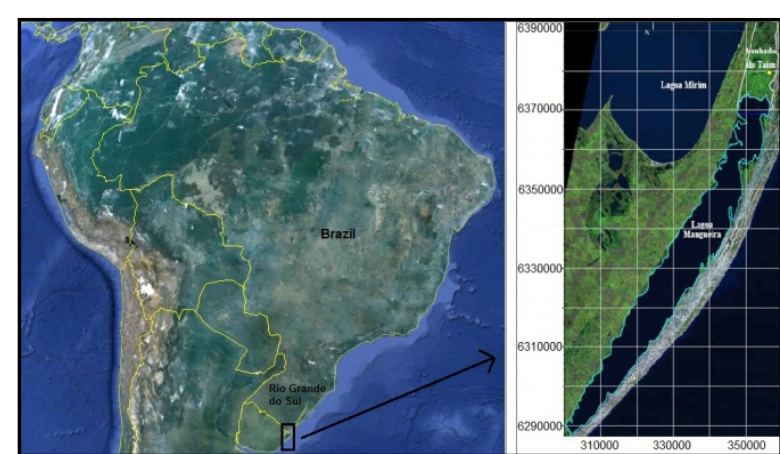

Figura 1. Área de estudo, Lagoa Mangueira, RS, Brasil.

\section{Metodologia}

As medidas de temperatura de superfície foram obtidas na região do infravermelho termal do produto MOD11(Temperatura de Superfície Terrestre e Emissividade). do sensor Moderate Resolution Imaging Spectroradiometer (MODIS) do satélite EOS-TERRA. As imagens foram adquiridas através do endereço eletrônico http://reverb.echo.nasa.gov/reverb nas datas das passagens das frentes frias dos do mês de dezembro e dos meses de inverno (junho/ julho) de 2006.

As observações de temperatura da superfície terrestre fornecidas pelo produto MOD11 do sensor MODIS (na resolução de $1 \mathrm{~km}$ ) são calculadas a partir do algoritmo "generalized split-window" (WAN e DOZIER, 1996), o qual assume um conhecimento a priori da emissividade. As medidas de emissividades das bandas 31(10,78 a 11,28 $\mu \mathrm{m})$ e 32(11,77 a 12,27 $\mu \mathrm{m})$ Segundo Wan \& Dozier (1996) a estimativa da temperatura de superfície através do algoritmo generalized split-window do sensor MODIS é dada por:

$$
T_{S}=\left(A_{1}+A_{2} \frac{1-\varepsilon}{\varepsilon}+A_{3} \frac{\Delta \varepsilon}{\varepsilon^{2}}\right) \frac{T_{31}+T_{32}}{2}+\left(B_{1}+B_{2} \frac{1-\varepsilon}{\varepsilon}+B_{3} \frac{\Delta \varepsilon}{\varepsilon^{2}}\right)\left(T_{31}-T_{32}\right)+C
$$

na qual, a temperatura da supefície terrestre, $T_{31}$ e $T_{32}$ são as temperaturas de brilho nas banda 31 e 32 do MODIS; $\varepsilon=0,5\left(\varepsilon_{31}+\varepsilon_{32}\right)$ e $\Delta \varepsilon=\varepsilon_{31}-\varepsilon_{32}$ são as emissividades da superfície nas bandas 31 e 32 do MODIS; C, A1, A2, A3, B1, B2, B3 são os coeficientes de regressão. Para a observação da variação de temperatura na lagoa Mangueira foi traçado um perfil longitudinal $(\mathrm{PL})$ na direção sul-norte nas imagens de temperatura do sensor MODIS O posicionamento do perfil PL na imagem da figura 2 é mostrado na Tabela 1.

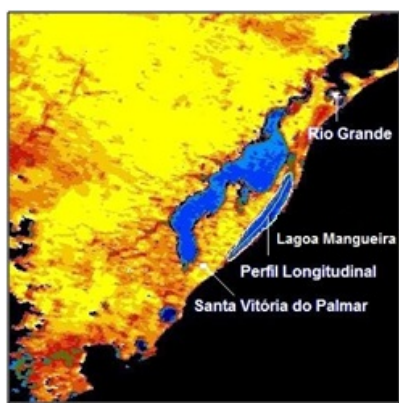

Figura 2. Imagem do sensor MODIS e do PL de temperatura de superfície na Lagoa Mangueira.
Tabela 1 - Posicionamento dos pontos do perfil PL de temperatura de superfície traçado na lagoa Mangueira.

\begin{tabular}{|c|c|c|c|c|}
\hline \multirow{2}{*}{ PL } & \multicolumn{2}{|c|}{ Ponto Inicial } & \multicolumn{2}{c|}{ Posição Final } \\
\cline { 2 - 5 } & Latitude & Longitude & Latitude & Longitude \\
\cline { 2 - 5 } & $33^{\circ} 30^{\prime} 40.2^{\prime \prime} \mathrm{S}$ & $53^{\circ} 6^{\prime} 41.2^{\prime \prime} \mathrm{W}$ & $32^{\circ} 35^{\prime} 10.5^{\prime \prime} \mathrm{S}$ & $52^{\circ} 31^{\prime} 39.9^{\prime \prime} \mathrm{W}$ \\
\hline
\end{tabular}

Os dados meteorológicos da área de estudo foram extraídos da Estação Meteorológica de Observação de Superfície Convencional localizada em Santa Vitória do Palmar (33³1'58.00"S; $53^{\circ} 20^{\prime} 58.56$ "O), RS, que coleta medidas diárias de variáveis climáticas. Neste estudo considera-se a velocidade do vento $(\mathrm{m} / \mathrm{s})$, a sua direção e a temperatura ambiente, $\operatorname{TA}\left({ }^{\circ} \mathrm{C}\right)$. Estas variáveis são coletadas em três horários distintos, $12 \mathrm{~h}, 18 \mathrm{~h}$ e $24 \mathrm{~h}$. As datas das passagens das frentes fria foram extraídas através do endereço eletrônico http://climanalise.cptec.inpe.br/ rclimanl/boletim/index0 806.shtm, para cada período estudado. Foram selecionados três sistemas frontais durante o mês de dezembro de 2005 , dois no mês de junho/2006 e um no mês de julho/2006 que passaram na região sul do Brasil. A influência destes sistemas frontais teve a seguinte duração, expressa em intervalos de dias, Tabela 2:

Tabela 2 - Período de duração das frentes frias.

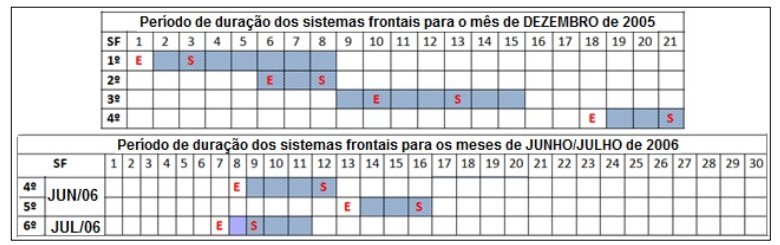

SF = Sistema Frontal, $\mathrm{E}=$ Entrada do sistema frontal em Santa Vitória do Palmar e S = Saída do sistema frontal em Santa Vitória do Palmar

As imagens do sensor MODIS foram selecionadas com base na cobertura de nuvens sobre a lagoa Mangueira. As imagens escolhidas representam dias de céu claro. Selecionou-se uma imagem antes da passagem da frente e uma imagem após a passagem da frente em Santa Vitória do Palmar, RS. A Tabela 3 e 4 indicam as frentes frias relacionadas com os dados micrometeorológicos para os dois períodos estudados.

Tabela 3. Datas das imagens MODIS correspondentes às frentes frias $e$ associadas aos dados micrometeorológicos de Santa Vitória do Palmar, RS nos mes de dezembro de 2005. 


\begin{tabular}{|c|c|c|c|c|c|c|c|}
\hline $\begin{array}{c}\text { DEZEMBRO } \\
2005\end{array}$ & $\begin{array}{c}\text { Data } \\
\text { Imagem } \\
\text { MODIS }\end{array}$ & $\begin{array}{c}\text { Hora } \\
\text { (h) }\end{array}$ & $\begin{array}{l}\text { TA } \\
\left({ }^{\circ} \mathrm{C}\right)\end{array}$ & $\begin{array}{c}\text { Vento } \\
(\mathrm{m} / \mathrm{s})\end{array}$ & $\begin{array}{c}\text { Direção } \\
\text { do } \\
\text { vento }\end{array}$ & $\begin{array}{c}\text { Média/dia } \\
\text { Vento } \\
(\mathbf{m} / \mathbf{s})\end{array}$ & $\begin{array}{c}\text { Média/dia } \\
\mathbf{T A}\left({ }^{\circ} \mathrm{C}\right)\end{array}$ \\
\hline \multirow{3}{*}{$1^{\mathrm{a}}$ Entrada } & \multirow{3}{*}{$01 / 12 / 05$} & 12 & 20,2 & 6,2 & $\mathrm{~S}$ & \multirow{3}{*}{5,2} & \multirow{3}{*}{17,7} \\
\hline & & 18 & 19,2 & 7,2 & $\mathrm{~S}$ & & \\
\hline & & 24 & 13,8 & 2,1 & $\mathrm{~S}$ & & \\
\hline \multirow{3}{*}{$1^{\text {aa Saída }}$} & \multirow{3}{*}{$03 / 12 / 05$} & 12 & 21,6 & 7,2 & $\mathrm{NE}$ & \multirow{3}{*}{8,0} & \multirow{3}{*}{22,8} \\
\hline & & 18 & 26,6 & 8,7 & $\mathrm{NE}$ & & \\
\hline & & 24 & 20,3 & 8,2 & $\mathrm{NE}$ & & \\
\hline \multirow{3}{*}{$2^{\mathrm{a}}$ Entrada } & \multirow{3}{*}{$06 / 12 / 05$} & 12 & 18 & 5,1 & SW & \multirow{3}{*}{5,8} & \multirow{3}{*}{16,6} \\
\hline & & 18 & 18,4 & 6,2 & $\mathrm{~S}$ & & \\
\hline & & 24 & 13,5 & 6,2 & SW & & \\
\hline \multirow{3}{*}{$2^{\mathrm{a}}$ Saída } & \multirow{3}{*}{$08 / 12 / 05$} & 12 & 21,2 & 8,2 & NW & \multirow{3}{*}{4,1} & \multirow{3}{*}{23,1} \\
\hline & & 18 & 26,6 & 1 & $\mathrm{SE}$ & & \\
\hline & & 24 & 21,5 & 3,1 & W & & \\
\hline \multirow{3}{*}{$3^{\mathrm{a}}$ Entrada } & \multirow{3}{*}{$10 / 12 / 05$} & 12 & 20,1 & 2,1 & SW & \multirow{3}{*}{1,7} & \multirow{3}{*}{20,2} \\
\hline & & 18 & 21,2 & 2,1 & $\mathrm{SE}$ & & \\
\hline & & 24 & 19,3 & 1 & $\mathrm{~N}$ & & \\
\hline \multirow{3}{*}{$3^{\text {a }}$ Saída } & \multirow{3}{*}{$13 / 12 / 05$} & 12 & 21,2 & 4,1 & SE & \multirow{3}{*}{5,8} & \multirow{3}{*}{20,7} \\
\hline & & 18 & 22 & 5,1 & E & & \\
\hline & & 24 & 19 & 8,2 & $\mathrm{E}$ & & \\
\hline
\end{tabular}

Tabela 4. Datas das imagens MODIS correspondentes às frentes frias e associadas aos dados micrometeorológicos de Santa Vitória do Palmar, RS nos meses de Junho e Julho de 2006.

\begin{tabular}{|c|c|c|c|c|c|c|c|}
\hline $\begin{array}{c}\text { JUNHO/JULHO } \\
2006\end{array}$ & $\begin{array}{c}\text { Data } \\
\text { Imagem } \\
\text { MODIS }\end{array}$ & $\begin{array}{c}\text { Hora } \\
\text { (h) }\end{array}$ & $\begin{array}{l}\text { TA } \\
\left({ }^{\circ} \mathbf{C}\right)\end{array}$ & $\begin{array}{l}\text { Vento } \\
(\mathrm{m} / \mathrm{s})\end{array}$ & $\begin{array}{c}\text { Direção } \\
\text { do } \\
\text { vento }\end{array}$ & $\begin{array}{c}\text { Média/dia } \\
\text { Vento } \\
(\mathbf{m} / \mathbf{s})\end{array}$ & $\begin{array}{l}\text { Média/dia } \\
\text { TA }\left({ }^{\circ} \mathbf{C}\right)\end{array}$ \\
\hline \multirow{3}{*}{$4^{\mathrm{a}}$ Entrada } & \multirow{3}{*}{$08 / 06 / 06$} & 12 & 19,3 & 5 & NW & \multirow{3}{*}{4,7} & \multirow{3}{*}{22,6} \\
\hline & & 18 & 27,8 & 7 & NW & & \\
\hline & & 24 & 20,8 & 2 & NW & & \\
\hline \multirow{3}{*}{$4^{\mathrm{a}}$ Saída } & \multirow{3}{*}{$12 / 06 / 06$} & 12 & 6,7 & 0 & - & \multirow{3}{*}{2,0} & \multirow{3}{*}{10,3} \\
\hline & & 18 & 14,8 & 3 & $\mathrm{~N}$ & & \\
\hline & & 24 & 9,4 & 3 & NE & & \\
\hline \multirow{3}{*}{$5^{\mathrm{a}}$ Entrada } & \multirow{3}{*}{$13 / 06 / 06$} & 12 & 10,6 & 2 & NW & \multirow{3}{*}{1,3} & \multirow{3}{*}{13,8} \\
\hline & & 18 & 17,3 & 1 & NW & & \\
\hline & & 24 & 13,7 & 1 & NW & & \\
\hline \multirow{3}{*}{$5^{\mathrm{a}}$ Saída } & \multirow{3}{*}{$16 / 06 / 06$} & 12 & 6,8 & 2 & SW & \multirow{3}{*}{1,7} & \multirow{3}{*}{8,1} \\
\hline & & 18 & 12 & 2 & SW & & \\
\hline & & 24 & 5,6 & 1 & $\mathrm{~S}$ & & \\
\hline \multirow{3}{*}{$6^{\mathrm{a}}$ Entrada } & \multirow{3}{*}{ 07/07/06 } & 12 & 13,6 & 2 & NW & \multirow{3}{*}{3,7} & \multirow{3}{*}{18,7} \\
\hline & & 18 & 24,6 & 3 & $\mathrm{NE}$ & & \\
\hline & & 24 & 18 & 6 & $\mathrm{NE}$ & & \\
\hline \multirow{3}{*}{$6^{\mathrm{a}}$ Saída } & \multirow{3}{*}{ 09/07/06 } & 12 & 11,2 & 8 & W & \multirow{3}{*}{6,7} & \multirow{3}{*}{12,3} \\
\hline & & 18 & 14,8 & 8 & $\mathrm{~W}$ & & \\
\hline & & 24 & 11 & 4 & $\mathrm{~W}$ & & \\
\hline
\end{tabular}

\section{Resultados}

As figuras 3a, 3b; 4a, 4b; 5a, 5b; 6a, 6b; 7a, $7 \mathrm{~b} ; 8 \mathrm{a}, 8 \mathrm{~b}$ representam as passagens iniciais e finais dos sistemas frontais que atingiram o sul do Brasil no mês de dezembro de 2005 e nos meses de junho e julho de 2006. Nesta figuras, a área da superfície da lagoa está evidenciada pelo traçado branco. As figuras, $9,10,11,12,13,14$, exibem o perfil PL de temperatura superficial da lagoa Mangueira (TSL).

Tabela 5 - - Estatísticas da temperatura do PL da lagoa Mangueira na entrada dos sistemas frontais no mês de dezembro de 2005 .

\begin{tabular}{|c|c|c|c|c|c|c|c|}
\hline \multicolumn{2}{|c|}{ SF } & $\begin{array}{c}\text { Dia } \\
\text { Imagem } \\
\text { MODIS }\end{array}$ & Máx & Mín & $\begin{array}{c}\bar{x} \\
\left({ }^{\circ} \mathbf{C}\right)\end{array}$ & S( $\left.{ }^{\circ} \mathbf{C}\right)$ & \multirow{2}{*}{$\mathbf{C V}(\%)$} \\
\hline \multirow{2}{*}{$1^{\mathbf{a}}$} & Entrada & $01 / 12 / 05$ & 23,39 & 22,55 & 23,00 & 0,24 & 1,04 \\
\cline { 2 - 8 } & Saída & $03 / 12 / 05$ & 21,63 & 20,55 & 21,09 & 0,25 & 1,18 \\
\hline \multirow{2}{*}{$2^{\mathbf{a}}$} & Entrada & $06 / 12 / 05$ & 21,91 & 21,03 & 21,41 & 0,18 & 0,84 \\
\cline { 2 - 8 } & Saída & $08 / 12 / 05$ & 20,79 & 19,83 & 20,15 & 0,22 & 1,09 \\
\hline \multirow{2}{*}{$3^{\text {a }}$} & Entrada & $10 / 12 / 05$ & 20,79 & 19,13 & 20,26 & 0,35 & 1,73 \\
\cline { 2 - 8 } & Saída & $13 / 12 / 05$ & 20,09 & 16,59 & 18,35 & 0,87 & 4,74 \\
\hline
\end{tabular}

Tabela 6 - - Estatísticas da temperatura do PL da lagoa Mangueira na entrada dos sistemas frontais nos meses de junho e julho de 2006.

\begin{tabular}{|c|c|c|c|c|c|c|c|c|}
\hline \multicolumn{2}{|c|}{ SF } & $\begin{array}{c}\text { Dia } \\
\text { Imagem } \\
\text { MODIS }\end{array}$ & $\begin{array}{c}\text { Número } \\
\text { de } \\
\text { pixels }\end{array}$ & Máx & Min & $\bar{x}\left({ }^{\circ} \mathbf{C}\right)$ & S( $\left({ }^{\circ} \mathbf{C}\right)$ & CV(\%) \\
\hline \multirow{2}{*}{$4^{\mathbf{a}}$} & Entrada & $08 / 06 / 06$ & 873 & 24,66 & 14,60 & 16,78 & 2,01 & 11,98 \\
\cline { 2 - 9 } & Saída & $12 / 06 / 06$ & 852 & 14,56 & 11,18 & 12,66 & 0,64 & 5,05 \\
\hline \multirow{2}{*}{$5^{\mathbf{a}}$} & Entrada & $13 / 06 / 06$ & 688 & 13,82 & 5,95 & 12,14 & 0,81 & 6,67 \\
\cline { 2 - 9 } & Saída & $16 / 06 / 06$ & 686 & 13,90 & 7,46 & 11,47 & 1,18 & 10,29 \\
\hline \multirow{2}{*}{$6^{\mathbf{a}}$} & Entrada & $07 / 07 / 06$ & 707 & 18,14 & 7,86 & 11,86 & 1,80 & 15,18 \\
\cline { 2 - 9 } & Saída & $09 / 07 / 06$ & 705 & 16,20 & 9,40 & 13,72 & 1,03 & 7,51 \\
\hline
\end{tabular}

$1^{\circ}$ Sistema Frontal
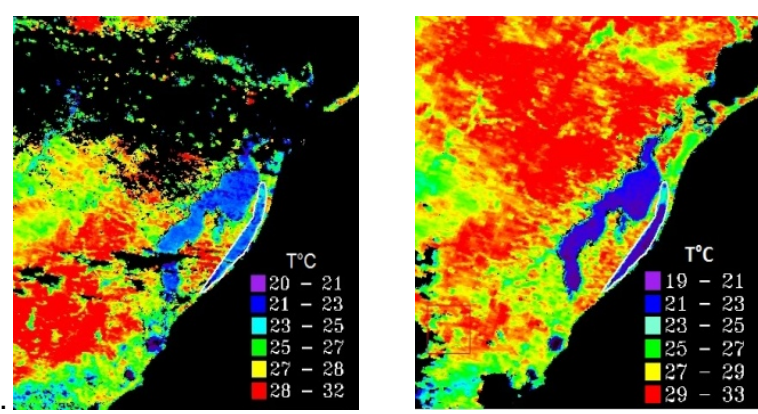

Figura 3 - Imagem (a) 01/12/05Imagem (b) 03/12/05

\section{$2^{\circ}$ Sistema Frontal}
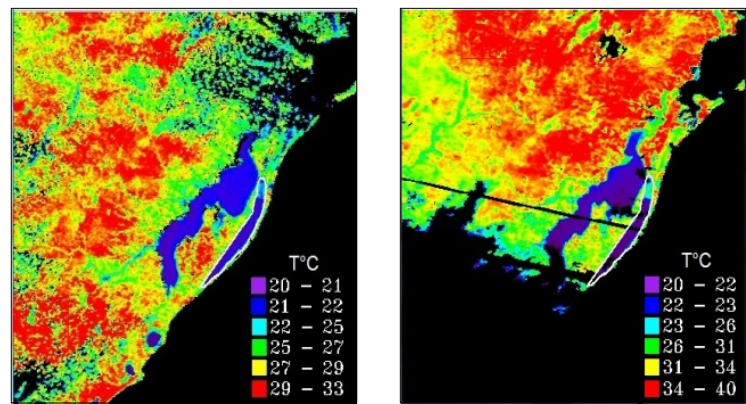

Figura 3 - Imagem (a) 06/12/05 Imagem (b) 08/12/05

\section{$3^{\circ}$ Sistema Frontal}
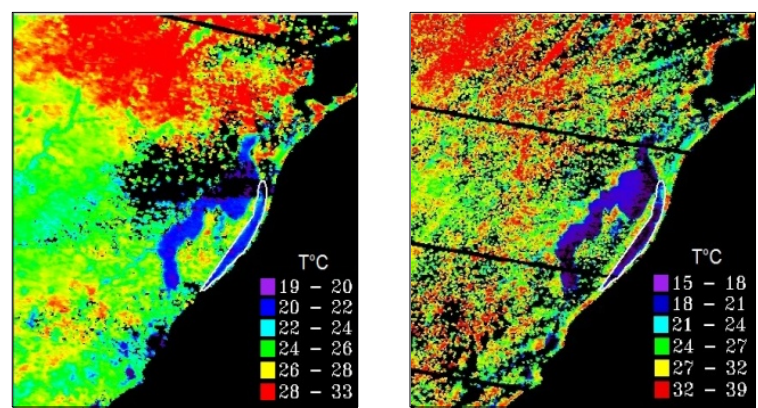

Figura 4 - Imagem (a) 10/12/05Imagem (b) 13/12/05 


\section{$4^{\circ}$ Sistema Frontal}
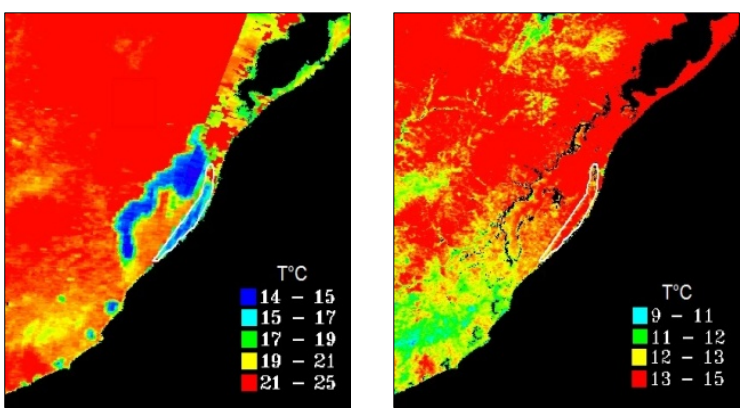

Figura 5 - Imagem (a) 08/06/06 Imagem (b) 12/06/06

$5^{\circ}$ Sistema Frontal
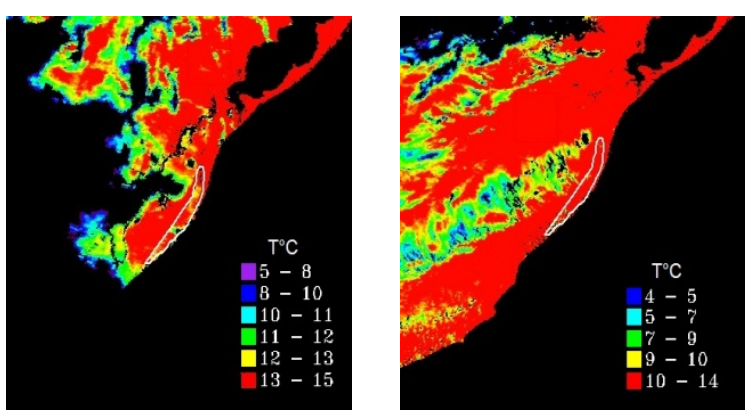

Figura 7 - Imagem (a) 13/06/06Imagem (b) 16/06/06

\section{$6^{\circ}$ Sistema Frontal}
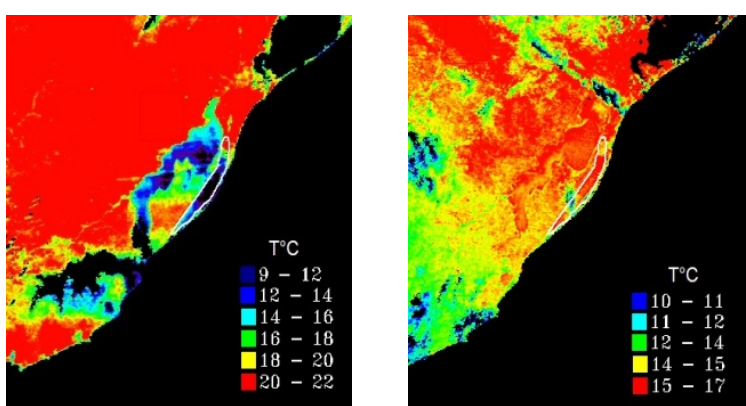

Figura 8 - Imagem (a) 07/07/06 Imagem (b) 09/07/06

\section{Perfis de Temperatura de dezembro/2005}

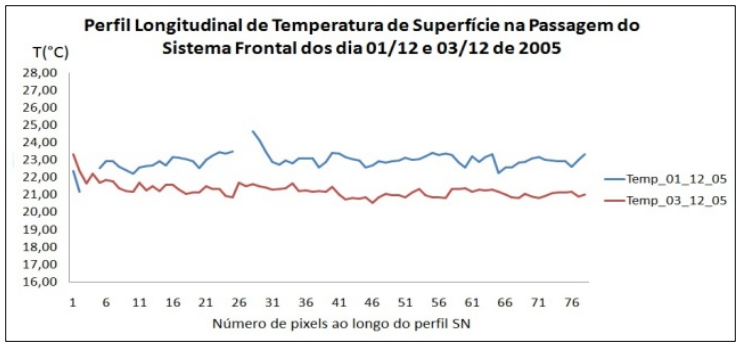

Figura 9 - PL na passagem do $1^{\circ} \mathrm{SF}$.

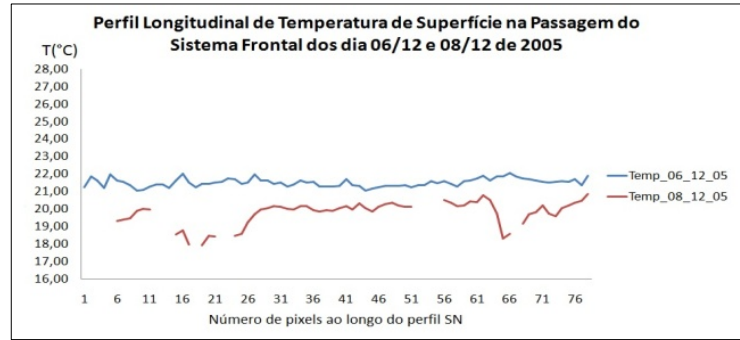

Figura $10-\mathrm{PL}$ na passagem do $2^{\circ} \mathrm{SF}$.

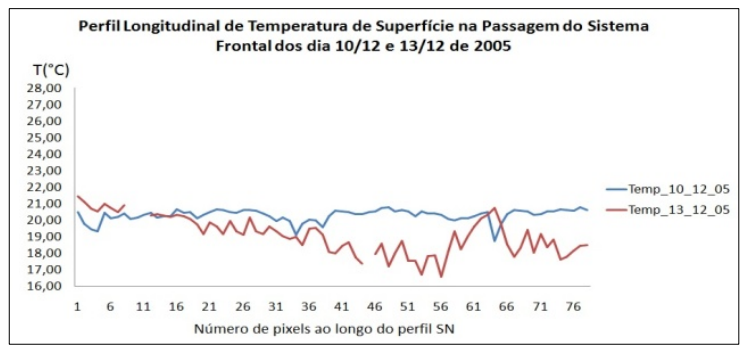

Figura 11 - PL na passagem do $3^{\circ} \mathrm{SF}$.

\section{Perfis junho/julho/2006}

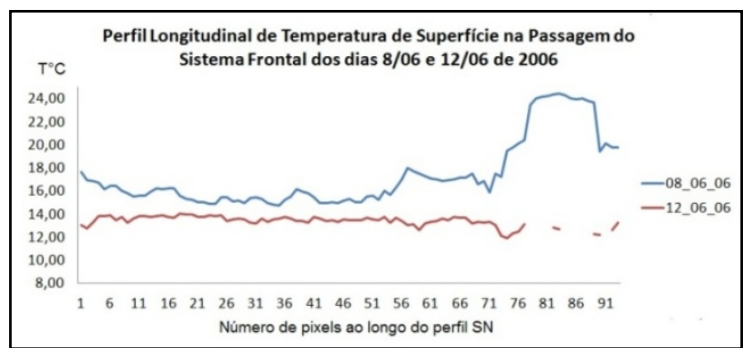

Figura 12 - Perfil L na passagem do $1^{\circ} \mathrm{SF}$.

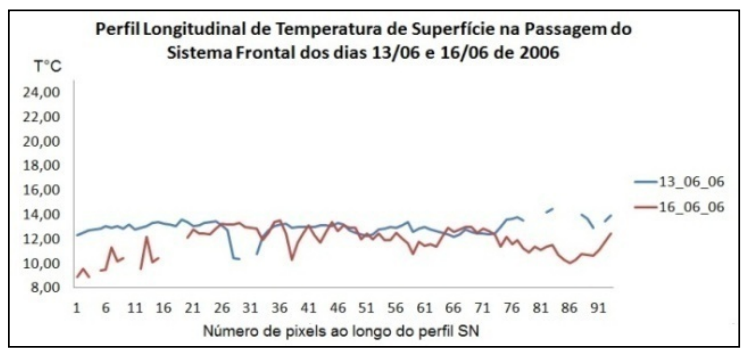

Figura 13 - Perfil L na passagem do $2^{\circ} \mathrm{SF}$.

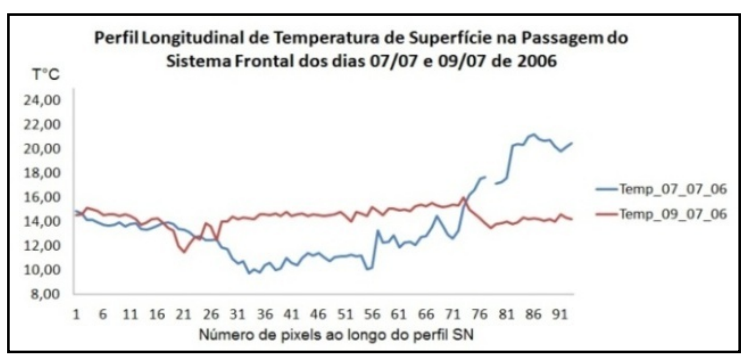

Figura 14 - Perfil L na passagem do $3^{\circ} \mathrm{SF}$. 


\section{Conclusões}

Os resultados evidenciaram que a passagem de um Sistema Frontal modifica a Temperatura Superficial da Lagoa (TSL). O processo de mudança da TSL está associado às interações meteorológicas da área de estudo. A investigação revela que existe uma relação entre os valores observados da temperatura superficial da lagoa com o vetor velocidade do vento (direção e magnitude) e com a $\operatorname{TA}\left({ }^{\circ} \mathrm{C}\right)$. Essa relação é evidenciada no para os dois períodos estudados.

As saídas das frentes frias provocam em dezembro uma diminuição média da TSL de $2^{\circ}$ e em junho e julho, uma diminuição média de $1^{\circ} \mathrm{C}$.

Em relação aos ventos, a presença de ventos fortes, com direções bem definidas, provoca grandes flutuações nas medidas da TSL. Estas advecções robustas, com magnitudes variando entre 5 e $8 \mathrm{~m} / \mathrm{s}$, são direcionalmente persistentes, ou seja, os ventos apresentam durante horas direções preferenciais consolidadas. Neste caso, as maiores flutuações de temperatura são conseqüência da turbulência da água, ocasionando assim maior instabilidade da TSL na superfície. Em dezembro estas flutuações são maiores do que nos meses de junho e julho, pois o vento apresenta maiores velocidades em períodos quentes, como pode ser observado através dos dados meteorológicos nas Tabelas 3 e 4 . Em dezembro, a maior variação da TSL é exibida na saída da terceira frente fria (Tabela 5). Esta passagem de frente está influenciada pela maior média de velocidade do vento $(5,8 \mathrm{~m} / \mathrm{s})$ em dezembro e $\mathrm{TA}\left({ }^{\circ} \mathrm{C}\right)$ elevada (média de $20,7^{\circ} \mathrm{C}$ ) na saída da frente fria.

Os resultados também revelam que, nos meses de junho e julho, para $\mathrm{TA}\left({ }^{\circ} \mathrm{C}\right)$ mais elevadas antes da passagem da frente fria, Tabelas 4 e 6 , ocorre maior variação da TSL, como pode ser observado na $4^{\mathrm{a}}$ e $6^{\mathrm{a}}$ frentes frias. Este escoamento de ar quente turbulento provocado pelo vento, logo acima da lagoa, transfere energia para a superfície da água gerando turbulência e ondas. Como consequência, esta turbulência na superfície da lagoa pode ser o processo físico responsável por provocar as flutuações observadas na TSL. A presente análise mostra que a análise comparativa de medidas remotas de temperatura superficial em lagoas, obtidas pelo sensor MODIS, constitui uma ferramenta viável e eficiente para estabelecer relações entre esta quantatidade e o campo de vento originado durante a passagem de sistemas frontais.

\section{Agradecimentos}

Os autores agradecem à Capes, CNPq e FAPERGS.

\section{Referências}

CLIMANÁLISE, Boletim de Monitoramento e Análise Climática, Vol. $20-\mathrm{n}^{\circ} 12$, dezembro/2005. Cachoeira Paulista, SP, Brasil, INPE/CPTEC, 1986. Publicação
Mensal. ISSN 0103-0019 CDU-555.5 http://climanalise.cptec.inpe.br/ rclimanl/boletim/index0 806.shtml acessado em setembro de 2012.

CLIMANÁLISE, Boletim de Monitoramento e Análise Climática, Vol. $21-\mathrm{n}^{\circ}$ 06, Junho/2006. Cachoeira Paulista, SP, Brasil, INPE/CPTEC, 1986. Publicação Mensal. ISSN 0103-0019 CDU-555.5 http://climanalise.cptec.inpe.br/ rclimanl/boletim/index0 806.shtml acessado em maio de 2013.

CLIMANÁLISE, Boletim de Monitoramento e Análise Climática, Vol. 21 - $\mathrm{n}^{\circ}$ 07, julho/2006. Cachoeira Paulista, SP, Brasil, INPE/CPTEC, 1986. Publicação Mensal. ISSN 0103-0019 CDU-555.5 http://climanalise.cptec.inpe.br/ rclimanl/boletim/index0 806.shtml acessado em maio de 2013.

DELANEY PJV (1965) Fisiografia e geologia da superfície da planície costeira do Rio Grande do Sul. Publ Esp Esc Geol Univ Fed Rio Grande Sul (Porto Alegre) 6:1-63.

MOHSENI O., STEFAN H.G. \& EATON J.G. (2003) Global warming and potential changes in fish habitat in U.S. streams. Climatic Change, 59, 389-409. NASA. Site de aquisição das Imagens do produto Mod11. Disponível em http://reverb.echo.nasa.gov/reverb. Acessado em dezembro de 2011.

TUNDISI, JG.; MATSUMURA-TUNDISI, T.; LUZIA, AP.; PASOERINI, MO.; CHIBA, W.; SEBASTIAN, NY. Cold fronts and reservoir limnology: an integrated approach towards the ecological dynamics of freshwater ecosystems. Brazilian Journal of Biology, v. 70, p. 815$824,2010$. 